\title{
Estimation of Multiple Point Sources for Linear Fractional Order Systems Using Modulating Functions
}

\author{
Zehor Belkhatir, Student Member, IEEE, and Taous-Meriem Laleg-Kirati,Senior Member, IEEE
}

\begin{abstract}
This paper proposes an estimation algorithm for the characterization of multiple point inputs for linear fractional order systems. First, using polynomial modulating functions method and a suitable change of variables the problem of estimating the locations and the amplitudes of a multi-pointwise input is decoupled into two algebraic systems of equations. The first system is nonlinear and solves for the time locations iteratively, whereas the second system is linear and solves for the input's amplitudes. Second, closed form formulas for both the time location and the amplitude are provided in the particular case of single point input. Finally, numerical examples are given to illustrate the performance of the proposed technique in both noise-free and noisy cases. The joint estimation of pointwise input and fractional differentiation orders is also presented. Furthermore, a discussion on the performance of the proposed algorithm is provided.
\end{abstract}

Index Terms - Linear fractional order systems, pointwise input, estimation, modulating functions method.

\section{INTRODUCTION}

$\mathbf{T}$ HE concept of fractional calculus stemmed in 1695 from a discussion between L'Hospital and Leibniz about the possibility of generalizing the order of classical derivatives to a non-integer order. Afterwards, in the twentieth century, differential equations involving fractional operators have gained considerable importance in modeling many real-world phenomena in different fields [1], [2], [3]. These fractional differential equations usually contain unknown differentiation orders, parameters and/or input to be estimated using available data. In particular, point source estimation problem has recently attracted the research community's attention for which interesting applications exist. Examples of applications include (a) the localization of groundwater point contaminant sources in fractional anomalous dispersion models [4] and (b) the characterization of the event-related type of neural activity in a fractional neurovascular model [5].

Several methods have been proposed in the literature for the estimation of parameters, and/or fractional differentiation orders of fractional order systems (FOSs). For example, a fractional version of the extended Kalman filter has been proposed in [6] to estimate the unknown parameters and the fractional differentiation orders of a discrete FOS; a simplified refined instrumental variable method with a Gauss-Newton

Z. Belkhatir and T. M Laleg-Kirati are with Computer, Electrical and Mathematical Sciences and Engineering Division, King Abdullah university of science and technology, KSA, email: \{zehor.belkhatir, taousmeriem.laleg\}@kaust.edu.sa approach was developed in [7] to identify the unknown parameters and the fractional differentiation orders. Moreover, an algebraic technique based on modulating functions has been proposed by Liu et al. [8] for parameters' identification. These functions have been defined and used for parameter identification of integer-order systems since the fifties [9]. Then, their properties have been studied in several papers [10], [11], [8], [12]. Recently, the modulating functions method has been combined with the first order Newton method to estimate both the coefficients and a single differentiation order of a fractional advection-dispersion equation [12]. This approach has been extended to the joint estimation of multiple fractional differentiation orders and parameters for fractional ordinary differential equations where the fractional derivative definition considers the history initialization function [13]. Modulating functions method offers several advantages comparing to state of the art methods, e.g., (i) it transfers the numerical fractional differentiation of the noisy output, avoiding numerical instability, to the analytical differentiation of the modulating functions, (ii) it does not require knowledge of the data at the boundaries which are in several situations unknown, and (iii) it is robust against noise on the output.

Unlike parameters' identification techniques, to the best of the authors' knowledge, input estimation for FOS is not well documented in the literature especially for non-smooth types of input like pointwise input. An example of existing work includes the fractional version of Zak-Walcott sliding mode observer which has been introduced in [14] to estimate the input jointly with the pseudo-state of FOS. Recently, an algorithm based on modulating functions has been proposed in [13] to estimate smooth inputs jointly with the fractional differentiation orders. More recently, a high-order sliding mode observer has been proposed in [15] for the joint estimation of the pseudo-state and the input of commensurate linear FOSs. For non-smooth inputs, a technique has been proposed to reconstruct a source term with sparse structure for fractional diffusion equations [16]. Also, a backward method has been developed in [4] to localize a pointwise source term of fractional advection dispersion equation. These methods are application-specific and not too generic.

This paper proposes an efficient method for the characterization of multiple point sources using polynomial modulating functions method. The contributions of this paper are two-fold: (i) We propose a suitable change of variables which allows to transform the original pointwise input's identification problem into two decoupled systems of equations. The first system is 
nonlinear and solves for time locations, and the second system is linear and solves for the amplitudes. (ii) We provide closedform formulas for the special case of a single point input based on the derived systems of equations proposed in (i).

The remainder of the paper is organized as follows. Section II recalls definitions and concepts on fractional calculus and modulating functions. In section III, the estimation problem is stated. In section IV, the proposed algorithm is developed for multiple point inputs. Moreover, closed-form formulas are derived for the case where the input is a single point. Section $\mathrm{V}$ presents different numerical tests that show the performance of the proposed algorithm in both noise-free and noisy cases. Discussions and concluding remarks are given in Sections VI and VII.

\section{PRELIMINARY}

In this section, background notions on fractional derivative and modulating functions are presented.

\section{A. Fractional derivative}

In the last few years, considerable interest has been given to the issue of initialization of FOS. This concept of initialization allows to define unique solutions [17], [18]. For this reason, in this work, we use the following initialized definition of fractional derivative[19].

Definition 1: The $\alpha^{\text {th }}$ Rieman-Liouville (RL) initialized fractional derivative of a function $f(t)$ is defined as in [19],

$$
D_{t}^{\alpha} f(t)=d_{t}^{\alpha} f(t)+\Psi\left(f_{0},-p,-t_{0}, 0, t\right), \quad t>0,
$$

with

$$
\left\{\begin{array}{l}
d_{t}^{\alpha} f(t)=\frac{\mathrm{d}^{n}}{\mathrm{~d}^{n}} \frac{1}{\Gamma(p)} \int_{0}^{t}(t-\tau)^{p-1} f(\tau) \mathrm{d} \tau, \\
\Psi\left(f_{0},-p,-t_{0}, 0, t\right)=\frac{\mathrm{d}^{n}}{\mathrm{~d}^{n}} \frac{1}{\Gamma(p)} \int_{-t_{0}}^{0}(t-\tau)^{p-1} f_{0}(\tau) d \tau,
\end{array}\right.
$$

where $\alpha=n-p$ with $n \in \mathbb{N}$ and $p, \alpha \in \mathbb{R}_{+}^{*}$. The terms $d_{t}^{\alpha} f(t)$, and $\Psi\left(f_{0},-p,-t_{0}, 0, t\right)$ are the uninitialized $\alpha^{t h}$ order RL derivative and its corresponding initialization function, respectively, $\Gamma($.$) is the standard Gamma function$ and $f_{0}(t)$ is the initialization history function defined for $t \in\left[-t_{0}, 0\right], t_{0} \in \mathbb{R}_{+}^{*}$. This definition assumes that the historyfunction for $t \in\left(-\infty,-t_{0}\right)$ is zero.

Remark 1: The initialized Caputo derivative is proposed in [20] for general fractional differentiation order. This definition has been shown to be equivalent to the initialized RL derivative for a constant initialization history function, i.e., $f_{0}(t)=b \in \mathbb{R}, \forall t \in\left[-t_{0}, 0\right]$. Without loss of generality, we will consider in this work the definition of the initialized RL fractional derivative for constant history functions.

\section{B. Modulating functions}

Definition 2: [21] A function $\phi(t)$, defined on $[0, T]$, is a modulating function of order $n$ if it satisfies the following properties,

(P1) $\phi \in C^{n}([0, T])$;
$(P 2) \phi^{(i)}(0)=\phi^{(i)}(T)=0, i=0,1, \ldots, n-1 ;$

where $C^{n}([0, T])$ denotes the space of $n$ times differentiable functions over $[0, T]$.

The modulating function $\phi(t)$ of order $n$ satisfies the following properties [22],

(P3) $D_{t}^{\alpha} \phi(t)$ exists, $\quad \forall 0 \leqslant \alpha \leqslant n$;

(P4) $\left.D_{t}^{\alpha} \phi(t)\right|_{t=0}=0 ; \quad \forall 0 \leqslant \alpha \leqslant n$.

A generalization of an integration by parts-like formula for uninitialized RL fractional derivative has been proposed in [13] for the case of constant initialization function, and is recalled in the following lemma.

Lemma 1: Let's consider a function $f(t)$ and let $\phi(t)$ be a modulating function of order $n$ with $n \in \mathbb{N}^{*}$. Assuming that the $\alpha^{t h}$ initialized RL fractional derivative of $f(t)$ exists, with $\alpha \in$ $\mathbb{R}_{+}^{*}$ and the constant initialization function $f_{0}(t)=b$, initialized from $t=-t_{0}, t_{0}>0$, then the following holds

$$
\begin{aligned}
& \int_{0}^{T} D_{t}^{\alpha} f(t) \phi(T-t) \mathrm{dt}=\int_{0}^{T} f(T-t) D_{t}^{\alpha} \phi(t) \mathrm{dt} \\
& \quad+\frac{b}{\Gamma(1-\alpha)} \int_{0}^{T} \phi(T-t)\left(t+t_{0}\right)^{-\alpha} \mathrm{dt}-b D_{t}^{\alpha-1} \phi(T) .
\end{aligned}
$$

Proof: The proof can be achieved using the Laplace transform, as performed in [8], and by making use of equations (44) and (14) of [19].

\section{Problem Formulation}

Let us consider the following FOS,

$$
y(t)+\sum_{i=1}^{N} a_{i} D_{t}^{\alpha_{i}} y(t)=u(t), \quad t \in[0, T],
$$

where $y(t)$ is the measured output, $u(t)$ is the unknown input supposed pointwise and given by the following equation

$$
u(t)=\sum_{i=1}^{L} \beta_{i} \delta\left(t-t_{i}\right)
$$

$\delta(t)$ denotes the Dirac-delta function and $\left\{t_{i}, \beta_{i}\right\}_{i=1}^{L}$ represent the time locations and amplitudes of the input, respectively. It is assumed that the time locations of the input are distinct. The parameters $\left\{a_{i}\right\}_{i=1}^{N} \in \mathbb{R}$ and the fractional differentiation orders $\left\{\alpha_{i}\right\}_{i=1}^{N} \in \mathbb{R}_{+}$are assumed to be known.

This paper focuses on solving the following estimation problem

\section{(Given the initialized FOS (3), characterize the unknown $\left\{\right.$ pointwise input, i.e., find estimates for $\left\{t_{i}, \beta_{i}\right\}_{i=1}^{L}$, from the measured output $y(t)$ with $t \in(0, T)$.}

\section{Main Results}

Let us define the following polynomial modulating functions,

$$
\phi_{m}(t)=\mu t^{M+q+1-m}(T-t)^{q+m},
$$

where $m=1,2, \ldots, M$ with $M$ is the total number of modulating functions, $q$ is an integer parameter and $\mu$ is a normalization factor. 
Let us also define the following change of variables

$$
\left\{\begin{array}{l}
\bar{\beta}_{i}=\mu \beta_{i}\left(T-t_{i}\right)^{M+q+1} t_{i}^{q}, \\
\theta_{i}=\frac{t_{i}}{T-t_{i}},
\end{array}\right.
$$

and denote

$$
\bar{\beta}=\left(\begin{array}{llll}
\bar{\beta}_{1} & \bar{\beta}_{2} & \ldots & \bar{\beta}_{L}
\end{array}\right)^{t r}, \theta=\left(\begin{array}{llll}
\theta_{1} & \theta_{2} & \ldots & \theta_{L}
\end{array}\right)^{t r},
$$

where $(t r)$ is the transpose operator.

\section{A. Characterization of multiple points input}

The following proposition introduces a new transformed nonlinear system of equations which solution provides estimate for the unknown vector $\theta$.

Proposition 1: Consider the FOS (3) initialized with the constant history function $f_{0}(t)=b, t \in\left[-t_{0}, 0\right]$, and let $\left\{\phi_{m}(t)\right\}_{m=1}^{2 L}$ be the set of polynomial modulating functions defined in (5). Then, the parameters $\left\{\theta_{i}\right\}_{i=1}^{L}$ solve the following nonlinear system of equations, $\forall i=1: L$,

$$
J_{i}(\theta)=\sum_{j=1}^{L} \sum_{k=1}^{L}(-1)^{k-1} \frac{\sigma_{L-k}^{j} \bar{b}_{j}}{\theta_{k}^{L-1+i} \prod_{\substack{v=1 \\ v \neq j}}^{L}\left(\theta_{v}-\theta_{j}\right)}-\bar{b}_{L+i}=0,
$$

where $\sigma_{m}^{j}$ denotes the m-th elementary symmetric function in the $L-1$ variables, $x_{i}$ for $i=1, \ldots, L$ and $i \neq j$, and which are defined in [23] as follows,

$$
\sigma_{m}^{j} \triangleq \sigma_{m}\left(\theta_{1}, \ldots, \theta_{j-1}, \theta_{j+1}, \ldots ., \theta_{L}\right)
$$

with $\sigma_{m} \triangleq \sigma_{m}\left(\theta_{1}, \theta_{2}, \ldots, \theta_{L}\right)=\sum_{1 \leq j_{1}<j_{2}<\ldots<j_{k} \leq L} \theta_{j_{1}} \theta_{j_{2}} \ldots . \theta_{j_{k}}$. and $\left\{\bar{b}_{m}\right\}_{m=1}^{2 L}$ are defined as follows

$$
\begin{gathered}
\bar{b}_{m}=\int_{0}^{T} \phi_{m}(T-t) y(t) \mathrm{dt}+\sum_{i=1}^{N}\left[a_{i} \int_{0}^{T} y(T-t) D_{t}^{\alpha_{i}} \phi_{m}(t) \mathrm{dt}\right. \\
\left.+\frac{b}{\Gamma\left(1-\alpha_{i}\right)} \int_{0}^{T} \frac{\phi_{m}(T-t)}{\left(t+t_{0}\right)^{\alpha_{i}}} \mathrm{dt}-b D_{t}^{\alpha_{i}-1} \phi_{m}(T)\right] .
\end{gathered}
$$

Proof:

First, we multiply (3) by the set of modulating functions $\left\{\phi_{m}(T-t)\right\}_{m=1}^{2 L}$ defined in (5), and we integrate over the time interval $[0, T]$. Then, using integration by parts formula (2), we obtain

$$
\begin{gathered}
\int_{0}^{T} \phi_{m}(T-t) y(t) \mathrm{dt}+\sum_{i=1}^{N}\left[a_{i} \int_{0}^{T} y(T-t) D_{t}^{\alpha_{i}} \phi_{m}(t) \mathrm{dt}+\right. \\
\left.\frac{b}{\Gamma\left(1-\alpha_{i}\right)} \int_{0}^{T} \frac{\phi_{m}(T-t)}{\left(t+t_{0}\right)^{\alpha_{i}}} \mathrm{dt}-b D_{t}^{\alpha_{i}-1} \phi_{m}(T)\right] \\
=\sum_{i=1}^{L} \beta_{i} \phi_{m}\left(T-t_{i}\right)=\sum_{i=1}^{L} \mu \beta_{i}\left(T-t_{i}\right)^{M+q+1-m} t_{i}^{q+m},
\end{gathered}
$$

Let us rewrite (11) in a compact form as follows

$$
\bar{b}_{m}=\sum_{i=1}^{L} \bar{\beta}_{i}\left(\theta_{i}\right)^{m}
$$

where $\bar{b}_{m}$ is the left hand side of equation (11) defined in (10) and $\bar{\beta}_{i}, \theta_{i}$ are defined in (6).
Second, we consider the set of modulating functions $\left\{\phi_{m}(t)\right\}_{m=1}^{L}$. Thus, equation (12) can be written as a linear system of equations in the unknown vector $\bar{\beta}$ as follows

$$
A(\theta) \bar{\beta}=\operatorname{col}\left\{\bar{b}_{i}\right\}_{i=1}^{L},
$$

where $\operatorname{col}\left\{\bar{b}_{i}\right\}_{i=1}^{L} \in \mathbb{R}^{L}$ denotes a column vector with elements $\bar{b}_{i}$ and the matrix $A(\theta) \in \mathbb{R}^{L \times L}$ given as follows

$$
A(\theta)=\left(\begin{array}{cccc}
\theta_{1} & \theta_{2} & \ldots & \theta_{L} \\
\left(\theta_{1}\right)^{2} & \left(\theta_{2}\right)^{2} & \ldots & \left(\theta_{L}\right)^{2} \\
\vdots & \vdots & \vdots & \vdots \\
\left(\theta_{1}\right)^{L} & \left(\theta_{2}\right)^{L} & \ldots & \left(\theta_{L}\right)^{L}
\end{array}\right)
$$

The matrix $A$ is of Vandermonde-like type of order $L$. Its inverse exists since the time locations are assumed distinct. This allows solving the linear system of equations and obtaining

$$
\bar{\beta}=A^{-1}(\theta) \operatorname{col}\left\{\bar{b}_{i}\right\}_{i=1}^{L} .
$$

Third, we consider the set of modulating functions $\left\{\phi_{m}(t)\right\}_{m=L+1}^{2 L}$. From equation (12) and (15), we get the following nonlinear system of equations with respect to the unknown vector $\theta$

$$
\bar{A}(\theta) A^{-1}(\theta) \operatorname{col}\left\{\bar{b}_{i}\right\}_{i=1}^{L}=\operatorname{col}\left\{\bar{b}_{i}\right\}_{i=L+1}^{2 L},
$$

where the matrix $q(\theta) \in \mathbb{R}^{L \times L}$ is given as follows

$$
\bar{A}=\left(\begin{array}{cccc}
\left(\theta_{1}\right)^{L+1} & \left(\theta_{2}\right)^{L+1} & \ldots & \left(\theta_{L}\right)^{L+1} \\
\left(\theta_{1}\right)^{L+2} & \left(\theta_{2}\right)^{L+2} & \ldots & \left(\theta_{L}\right)^{L+2} \\
\vdots & \vdots & \vdots & \vdots \\
\left(\theta_{1}\right)^{2 L} & \left(\theta_{2}\right)^{2 L} & \ldots & \left(\theta_{L}\right)^{2 L}
\end{array}\right) .
$$

Using the closed form formula for the inverse of standard Vandemonde matrix [23] we can write explicitly the nonlinear system of equations (16) in terms of the unknowns $\left\{\theta_{i}\right\}_{i=1}^{L}$ as given in (8).

\section{B. Estimation procedure for the input's characteristics}

The estimation of the amplitudes and locations of the pointwise input follows from the result of Proposition 1. The nonlinear system of equations defined in Proposition 1 is given by the following equation

$$
J(\theta)=\left\{J_{i}(\theta)\right\}_{i=1}^{L}=0 .
$$

\section{First step:}

The use of global Newton method allows us to write the iterative update law (19) in order to solve the nonlinear system of equations (18) for the variable $\theta$.

$$
\theta^{k+1}=\theta^{k}+\lambda^{k} p^{k},
$$

where $\lambda^{k}$ is a nonuniform step size and

$$
p^{k}=-\left[J^{\prime}\left(\theta^{k}\right)\right]^{-1} J\left(\theta^{k}\right),
$$

with $J\left(\theta^{k}\right) \in \mathbb{R}^{L \times 1}$ and $J^{\prime}\left(\theta^{k}\right) \in \mathbb{R}^{L \times L}$ is the corresponding Jacobian matrix. The parameter $\lambda^{k}$ can be computed using the backtracking line search method [24]. 


\section{Second step:}

Once the Newton method converges, we get from equation (15) the estimate, $\hat{\bar{\beta}}$, of the variable $\bar{\beta}$ which is given as follows

$$
\hat{\bar{\beta}}=A^{-1}(\hat{\theta}) \operatorname{col}\left\{\bar{b}_{i}\right\}_{i=1}^{L},
$$

where $A(\hat{\theta})$ and $\bar{b}$ are defined in (14) and (10), respectively.

\section{Third step}

The estimates of the variables $\left\{t_{i}\right\}_{i=1}^{L}$ and $\left\{\beta_{i}\right\}_{i=1}^{L}$ (22), noted $\hat{t}_{i}$ and $\hat{\beta}_{i}$, are derived from the change of variables defined in (6).

$$
\left\{\begin{array}{l}
\hat{t}_{i}=T \frac{\hat{\theta}_{i}}{1+\hat{\theta}_{i}}, \\
\hat{\beta}_{i}=\frac{\hat{\bar{\beta}}_{i}}{\mu\left(T-\hat{t}_{i}\right)^{M+q+1} \hat{t}_{i}^{q}}
\end{array}\right.
$$

The main steps of the proposed algorithm are summarized in Table I.

TABLE I

STEPS OF THE PROPOSED ALGORITHM FOR POINTWISE INPUT'S CHARACTERIZATION.

Step 1: Initialize $k=0$, give an initial guess to $\theta^{0}$ and $\lambda^{0}=1$;

Step 2: Compute $J\left(\theta^{k}\right)$ using equation (18);

Step 3:

- If $\left.\| J\left(\theta^{k}\right)\right) \|_{2}<\varepsilon$, then stop and get the estimate $\hat{\theta}=\theta^{k}$,

- else,

(i) : compute $\lambda^{k}$ using any search line method. For the backtracking method, follow the following procedure

(i.1) define the merit function $m(\theta)=\frac{1}{2} J(\theta)^{\operatorname{tr}} J(\theta)$, and compute its gradient $\nabla_{\theta} m\left(\theta^{k}\right)$,

(i.2) compute $p^{k}$ defined in (20),

(i.3) while $\left[m\left(\theta^{k}+\lambda^{k} p^{k}\right)>m\left(\theta^{k}\right)+\bar{\alpha} \lambda^{k} \nabla_{\theta} m\left(\theta^{k}\right) p^{k}\right]$ $\lambda^{k}=n \lambda^{k-1}, \quad n<1$

$\{\bar{\alpha}, n$ are the algorithm parameters chosen by the user $\}$, end

(ii) : update $\theta^{k}$ using equation (19), update the number of iterations

$k=k+1$, and then return to step 2 ;

Step 4: Solve (21) using $\hat{\theta}$ derived in step 3 and get the estimates $\hat{\bar{\beta}}$;

Step 5: Derive the estimates for the time locations and the amplitudes of

the multiple points input using (22) and the estimates of steps 3 and 4.

\section{Special case: single-point input}

In this subsection, we provide the following corollary for the special case of a single point input.

Corollary 1: Consider the FOS (3) with a single point input, i.e., $L=1$, initialized with the constant history function $f_{0}(t)=b, t \in\left[-t_{0}, 0\right]$, and let $\left\{\phi_{m}(t)\right\}_{m=1}^{2}$ be the set of polynomial modulating functions defined in (5). Then, an exact characterization of the location and the amplitude of the pointwise input is given as follow

$$
\left\{\begin{array}{l}
t_{1}=T \frac{\bar{b}_{2}}{\bar{b}_{1}+\bar{b}_{2}}, \\
\beta_{1}=\frac{\bar{b}_{1}}{\phi_{1}\left(T-t_{1}\right)},
\end{array}\right.
$$

where $\bar{b}_{i}$ defined as in equation (10).

Proof: The proof follows the steps given in the previous subsection. In this case, the nonlinear equation (8) of Proposition 1 is

$$
J(\theta)=\frac{\bar{b}_{1}}{\theta_{1}}-\bar{b}_{2}=0
$$

which can be solved analytically. Then, the estimates (23) follow directly from equation (22).

Remark 2: In [13], a two-stage algorithm is proposed to estimate the parameters jointly with the fractional differentiation orders. In the same spirit, the algorithm proposed in this paper can be combined with any iterative technique to solve the problem of estimating the pointwise input jointly with the fractional differentiation orders in two stages. We do not include the derivations of technical details for the twostage algorithm in this paper. However, the reader can follow the same approach in [13] for such derivations.

\section{Numerical Results}

All the simulation results presented in this section assume that the initialization function $f_{0}(t)=0, \forall t \in(-\infty, 0]$. The analytical expression of the $\alpha^{\text {th }}$ RL fractional derivative of the polynomial modulating functions given in (5) is as follows

$$
D_{t}^{\alpha} \phi_{m}(t)=\sum_{k=0}^{q+m} c_{k} \frac{\Gamma(M+q-m+k+2)}{\Gamma(M+q-m+k+2-\alpha)} t^{M+q+1-m+k-\alpha},
$$

where $c_{k}=\mu\left(\begin{array}{c}q+m \\ k\end{array}\right)(-1)^{k} T^{q+m-k}$.

We also use the following Grünwald-Letnikov definition to implement numerically the fractional derivatives.

$$
D_{t}^{\alpha} f(t)=\lim _{h \rightarrow 0} \frac{1}{h^{\alpha}} \sum_{i=0}^{\lfloor t / h\rfloor} c_{i}^{(\alpha)} f(t-i h), \quad \alpha>0,
$$

where $h>0$ is the time step, $\lfloor$.$\rfloor is the floor operator, and$ $c_{i}^{(\alpha)}(i=0,1, \ldots)$ are the binomial coefficients which are recursively computed using the following formula

$$
c_{0}^{(\alpha)}=1, c_{i}^{(\alpha)}=\left(1-\frac{1+\alpha}{i}\right) c_{i-1}^{(\alpha)} .
$$

The Dirac-delta function is approximated numerically by the following Gaussian function

$$
\delta\left(t-t_{i}\right) \approx \frac{1}{h \sqrt{2 \pi}} \exp \left[-\frac{\left(t-t_{i}\right)^{2}}{2 h^{2}}\right],
$$

which satisfies the property of unit area under the curve, i.e., $\int_{-\infty}^{+\infty} \delta(t) d t=1$, for $h$ small. In all the numerical simulations, the time step is $h=10^{-2}$.

As an application to the developed algorithm, we propose to localize inputs with very short support for a fractional neurovascular coupling model [5]. This type of input corresponds to a fast event-related neural activity which is approximated mathematically by a Dirac-delta function. The neurovascular model is given as follows

$$
D_{t}^{\alpha_{1}} F(t)+k D_{t}^{\alpha_{2}} F(t)+\gamma F(t)=u(t), t \in[0,10],
$$


where $1 \leq \alpha_{1} \leq 2,0 \leq \alpha_{2} \leq 1$ are the fractional differentiation orders, $F(t)$ is the cerebral blood flow and $u(t)$ is the pointwise neuronal activity. The constants $k$ and $\gamma$ are the flow signal decay rate and the flow-dependant elimination constant, respectively. Their values are known $k=0.65, \gamma=0.41$ as given in [25]. The values of the fractional differentiation orders are chosen to be: $\alpha_{1}=1.8$ and $\alpha_{2}=0.6$.

In the noisy case, we added a zero-mean white Gaussian noise $e$ with a relative noise level $\frac{\|e\|_{2}}{\left\|F_{\text {noise-free }}\right\|_{2}} \times 100(\%)$ [26, pp. 50]. Moreover, the results given in the following represent a sample from one realization. Monte Carlo simulations have been tested as well and the overall results and conclusions remain the same.

\section{A. Case 1: characterization of single point input}

In this case the input is set as $u_{1}(t)=\beta_{0} \delta\left(t-t_{0}\right)$, with $\beta_{0}=3$ and $t_{0}=8$. Figure 1 shows the input $u_{1}(t)$ and its corresponding output $F_{1}(t)$. The estimated values for the time location and the amplitude are summarized in Table II.
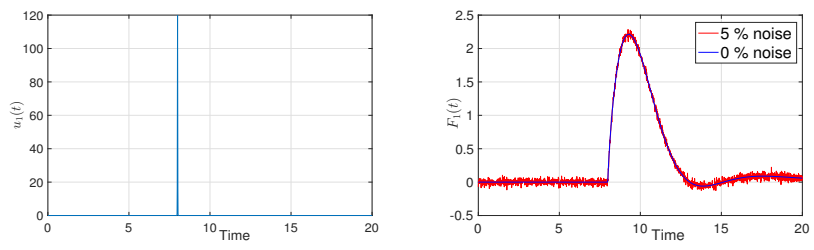

Fig. 1. Single point input (left) and its corresponding output (right).

TABLE II

RELATIVE ERRORS OF THE ESTIMATED PARAMETERS FOR SINGLE POINT INPUT.

\begin{tabular}{|l||c|c|}
\hline Noise level Relative error (\%) & $\left|\frac{t_{0}-\hat{t}_{0}}{t_{0}}\right| \times 100$ & $\left|\frac{\beta_{0}-\hat{\beta}_{0}}{\beta_{0}}\right| \times 100$ \\
\hline \hline $0 \%$ noise & 0.011 & 0.036 \\
\hline $5 \%$ noise & 0.075 & 0.1197 \\
\hline $10 \%$ noise & 0.5395 & 0.276 \\
\hline $30 \%$ noise & 0.3753 & 0.3811 \\
\hline
\end{tabular}

Table II illustrates the good performance of the proposed methodology to characterize a single point input.

\section{B. Case 2: characterization of two points input}

In this case the input is set as $u_{2}(t)=\beta_{1} \delta\left(t-t_{1}\right)+\beta_{2} \delta\left(t-t_{2}\right)$, with $\quad \beta_{1}=3, t_{1}=3$, $\beta_{2}=0.5$ and $t_{2}=7$. The nonlinear system of equations provided in Proposition 1, is derived and given as follows

$$
J(\theta)=\left(\begin{array}{c}
-\theta_{1} \theta_{2} \bar{b}_{1}+\left(\theta_{1}+\theta_{2}\right) \bar{b}_{2}-\bar{b}_{3} \\
-\theta_{1} \theta_{2}\left(\theta_{1}+\theta_{2}\right) \bar{b}_{1}+\left(\theta_{1}^{2}+\theta_{1} \theta_{2}+\theta_{2}^{2}\right) \bar{b}_{2}-\bar{b}_{4}
\end{array}\right)
$$

and for which the Jacobian matrix can be easily computed.

Figure 2 shows the input $u_{2}(t)$ and its corresponding output $F_{2}(t)$. The estimated values for the time locations and the amplitudes are summarized in Table III.

Table III shows that for the selected initial guess $\theta^{0}=0.5 \theta_{\text {real }}$, where $\theta_{\text {real }}$ is the true value, $\lambda^{k}=1$ is sufficient
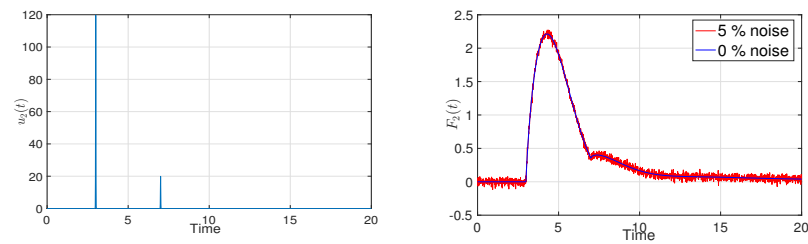

Fig. 2. Two points input (left) and its corresponding output (right).

TABLE III

RELATIVE ERRORS OF THE ESTIMATED PARAMETERS FOR TWO-POINT INPUT, WITH $\lambda^{k}=1$ AND $\theta^{0}=0.5 \theta_{\text {real }}$.

\begin{tabular}{|l||c|c|c|c|}
\hline Noise level & $t_{1}$ & $\beta_{1}$ & $t_{2}$ & $\beta_{2}$ \\
\hline \hline $0 \%$ noise & 0.1404 & 0.2688 & 0.237 & 1.2034 \\
\hline $5 \%$ noise & 0.8899 & 0.0741 & 1.224 & 2.667 \\
\hline $10 \%$ noise & 1.948 & 1.0528 & 2.301 & 4.6451 \\
\hline $30 \%$ noise & 11.9025 & 21.553 & 7.7798 & 20.9949 \\
\hline
\end{tabular}

to obtain good results. However, for further values of $\theta_{0}$ the algorithm becomes sensitive to the initial guesses and the adaptation of $\lambda^{k}$ is necessary. Table IV shows the estimation results for an initial condition $\theta^{0}=5 \theta_{\text {real }}$ and for which a backtracking line search method is used to update the step size. It is clear from those reported results that the global Newton method converges to the true values of the time locations and amplitudes even with a noisy output.

TABLE IV

RELATIVE ERRORS OF THE ESTIMATED PARAMETERS FOR TWO-POINT INPUT USING GLOBAL NEWTON METHOD AND $\theta^{0}=5 \theta_{\text {real }}$.

\begin{tabular}{|l||c|c|c|c|}
\hline Roise level & $t_{1}$ & $\beta_{1}$ & $t_{2}$ & $\beta_{2}$ \\
\hline \hline $0 \%$ noise & 0.1243 & 0.3 & 0.214 & 1.1795 \\
\hline $5 \%$ noise & 0.8792 & 0.0509 & 1.2212 & 2.6535 \\
\hline $10 \%$ noise & 1.9396 & 1.0401 & 2.2973 & 4.6282 \\
\hline $30 \%$ noise & 11.5572 & 20.1916 & 7.74988 & 20.7331 \\
\hline
\end{tabular}

C. Case 3: characterization of single point input jointly with the estimation of the fractional differentiation orders

The input is fixed as $u_{3}(t)=u_{1}(t)$. In this case, we consider a two-stage algorithm detailed in [13]. The first stage estimates the time location and the amplitude as in Corollary 1, however, the second stage solves a nonlinear system for the fractional differentiation orders using first-order Newton method. The results of the estimated parameters are shown in Table V.

TABLE V

RELATIVE ERRORS OF THE ESTIMATED PARAMETERS FOR SINGLE POINT INPUT AND UNKNOWN FRACTIONAL DIFFERENTIATION ORDERS USING GLOBAL NEWTON METHOD AND $\alpha^{0}=0.8 \alpha_{\text {real }}$.

\begin{tabular}{|c|c|c|c|c|}
\hline $\begin{array}{ll}\text { Noise level } & \text { Relative error (\%) } \\
\end{array}$ & $t_{0}$ & $\beta_{0}$ & $\alpha_{1}$ & $\alpha_{2}$ \\
\hline $0 \%$ noise & 0.2203 & 0.8046 & 0.0573 & 1.5379 \\
\hline $5 \%$ noise & $8.36 \times 10^{-2}$ & 0.6538 & $4.93 \times 10^{-2}$ & 0.7834 \\
\hline $10 \%$ noise & $3.8 \times 10^{-2}$ & 0.5746 & 0.1651 & 0.1735 \\
\hline $30 \%$ noise & 0.5896 & $1.28 \times 10^{-2}$ & 0.5938 & 2.7722 \\
\hline
\end{tabular}




\section{DISCUSSIONS}

From the numerical study of the previous three cases, the following observations are worth being emphasized:

- The characterization of a single input is very robust. Even with high noise level, we were able to estimate the input's characteristics efficiently. This can be explained by the exact analytic characterization obtained in this case.

- When the number of unknown increases, for instance, the case of two points input, the algorithm is sensitive to the initial guess of the unknowns. However, using a backtracking search method helped us to overcome this problem in the case of a two-point input.

- When the two sources of the concentrated input are relatively close, the algorithm fails to detect them accurately. The algorithm works well for distinct sources. This is due to the ill-conditioning of the Vandermonde-like matrix. In fact, the inverse of such type of matrices is defined if the time locations are distinct. The case of relatively close time locations leads to an ill-conditioned matrix, and hence its inverse is numerically unstable.

- In the case of unknown input and fractional differentiation orders, the algorithm is more sensitive to the initial guess value. For this case, even with the use of backtracking search method, the algorithm had difficulties in estimating all the unknown parameters accurately.

- The normalization parameter $\mu$ helps in alleviating some numerical issues that may happen when inverting the Jacobian matrices of the nonlinear systems defined in Proposition 1 and the one that arises from the second stage of case 3. In our simulations, we chose this parameter to be the inverse of the second or infinity norms of the standard polynomial modulating functions.

\section{CONCLUSION}

In this paper, we proposed an algorithm for the joint estimation of the time locations and amplitudes of multiple points input using an appropriate change of variables and polynomial modulating functions. The proposed change of variables allowed to transform the investigated estimation problem into two systems of equations. The first system is nonlinear and solves for the time locations. However, the second system is linear and solves for the input's amplitudes. To test the accuracy of the proposed algorithm, numerical examples have been provided both in noise-free and noisy cases. The obtained results have been discussed and some guidelines in the implementation of the algorithm have been provided.

\section{REFERENCES}

[1] R. Magin, Fractional calculus in bioengineering. Begell House Publisher, Inc, 2006.

[2] C. Ionescu, "Emerging tools in engineering: Fractional order ladder impedance models for respiratory and neural systems," IEEE journal on emerging and selected topics in circuits and systems, vol. 3, no. 3, pp. $425-431,2013$.

[3] A. A. Kilbas, H. M. Srivastava, and J. J. Trujillo, Theory and applications of fractional differential equations, Volume 204 (North-Holland Mathematics Studies). New York, NY, USA: Elsevier Science Inc., 2006.
[4] Y. Zhang, M. Meerschaert, and R. Neupauer, "Backward fractional advection dispersion model for contaminant source prediction," Water Resources Research, vol. 52, pp. 2462-2473, 2016.

[5] Z. Belkhatir and T. M. Laleg-Kirati, "Fractional dynamical model for neurovascular coupling," 36th Annual International Conference of the IEEE Engineering in Medicine and Biology Society (EMBC), pp. 49164919, 2014.

[6] D. Sierociuk and A. Dzielinski, "Fractional kalman filter algorithm for the states parameters and order of fractional system estimation," International Journal of Applied Mathematics and Computer Science, vol. 16, no. 1, pp. 129-140, 2006.

[7] S. Victor, R. Malti, H. Garnier, and A. Oustaloup, "Parameter and differentiation order estimation in fractional models," Automatica, vol. 49, pp. 926-935, 2013.

[8] D. Y. Liu, T. M. Laleg-Kirati, O. Gibaru, and W. Perruquetti, "Identification of fractional order systems using modulating functions method," in the American Control Conference, Washington, DC, USA,, 2013.

[9] M. Shinbrot, "On the analysis of linear and nonlinear dynamic systems from transient-response data, national advisory committee for aeronautics naca," Technical Note 3288, Washington, Tech. Rep.,, 1954.

[10] T. Co and S. Ungarala, "Batch scheme recursive parameter estimation of continuous-time system using the modulating functions method," Automatica, vol. 33, pp. 1185-1191, 1997.

[11] T. Janiczek, "Generalization of modulating functions method in the fractional differential equations," Bulletin of the academy of Sciences, vol. 58, no. 4, pp. 593-599, 2010.

[12] A. Aldoghaither, D. Y. Liu, and T. M. Laleg-Kirati, "Modulating functions based algorithm for the estimation of the coefficients and differentiation order for a space-fractional advection-dispersion equation," SIAM Journal on Scientific Computing, vol. 37, no. 6, pp. A2813A2839, 2015.

[13] Z. Belkhatir and T. M. Laleg-Kirati, "Parameters and fractional differentiation orders estimation for linear continuous-time noncommensurate fractional order systems," submitted, 2016. [Online]. Available: http://hdl.handle.net/10754/623960

[14] S. C. Lee, Y. Li, Y. Q. Chen, and H. S. Ahn, " $H_{\infty}$ and sliding mode observers for linear time-invariant fractional-order dynamic systems with initial memory effect," Journal of Dynamic Systems, Measurement and Control, vol. 136, 2014.

[15] Z. Belkhatir and T. Laleg-Kirati, "High-order sliding mode observer for fractional commensurate linear systems with unknown input," Automatica, vol. 82, pp. 209-217, 2017.

[16] Z. Ruan, Z. Yang, and X. Lu, "An inverse source problem with sparsity onstraint for the time-fractional diffusion equation," Advances in Applied Mathematics and Mechanics, vol. 8, no. 1, pp. 1-18, 2016.

[17] Kai Diethelm, The analysis of fractional differential Equations. Lecture Notes in Mathematics, Springer, 2010.

[18] T. Hartley, C. Lorenzo, J. Trigeassou, and N. Maamri, "Equivalence of history-function based and infinite-dimensional-state initializations for fractional-order operators," Journal of Computational and Nonlinear Dynamics, vol. 8, pp. 1-7, 2013.

[19] C. Lorenzo and T. Hartley, "Initialization of fractional-order operators and fractional differential equations," Journal of Computational and Nonlinear Dynamics, vol. 3, pp. 1-9, 2008.

[20] C. Lorenzo, T. Hartley, and J. Adams, "Time-varying initialization and corrected laplace transform of caputo derivative," 6th Workshop on Fractional Differentiation and Its Applications, pp. 161-166, 2013.

[21] H. Preising and D. Rippin, "Theory and application of the modulating function i. review and theory of the method and theory of the spline-type modulating functions," Computers \& Chemical Engineering, vol. 17, no. 1, pp. 1-16, 1993.

[22] I. Podlubny, Fractional differential equations. New York: Academic Press, 1999.

[23] W. Gautschi, "On inverses of vandermonde and confluent vandermonde matrices," Numerische Mathematik, vol. 4, pp. 117-123, 1962.

[24] J. Nocedal and S. Wright, Numerical Optimization. USA: Springer series in operation research and financial engineering, 2000.

[25] K. Friston, L. Harrison, and W. Penny, "Dynamic causal modeling," NeuroImage, vol. 19, pp. 1273-1302, 2003.

[26] C. Hansen, Discrete inverse problems: Insight and algorithms. Society for Industrial and Applied Mathematics Philadelphia, 2010. 